\title{
Zur Technik der Entnahme ungestörter Grossproben von Seesediment: ein verbessertes Bohrlot
}

\author{
Von H. AmbüHL und H. BüHRER \\ Eidg. Technische Hochschule Zürich \\ Institut für Gewässerschutz und Wassertechnologie ${ }^{1}$ ) \\ Manuskript eingegangen am 20. Dezember 1974
}

\section{SUMMARY}

A new sampling device for the extraction of completely undisturbed samples from lake sediments is described. The core has a diameter of $110 \mathrm{~mm}$ and a length of $60 \mathrm{~cm}$ or more. Technical prerequisites: sampling tube without interfering structure and with a very sharp end. The valve (to retain the sample) is designed so that water can flow out of the tube when the device is thrust into the sediment but is absolutely tight after sampling. If the sediment is compact (clay-like) sampling can be carried out without closing the tube at the base. There is an automatic closing mechanism for material with a high water content as well as an apparatus for subsequent preparation of the sample.

\section{Einleitung}

Mit einem 1969 beschriebenen Gerät [1] zur Entnahme von Proben mit einem Kerndurchmesser von $112 \mathrm{~mm}$ ist es prinzipiell möglich, ein ziemlich ungestörtes Material zu gewinnen, dessen Qualität für manche Bedürfnisse, insbesondere stratigraphische, ausreicht. Das Gerät besitzt einen den üblichen Schlammgreifern nachgebildeten Stech- und Schliessfuss, dessen Form in der Weise modifiziert ist, dass er nur minimalen Raum beansprucht und beim Einstich lediglich den kleinsten theoretisch möglichen Weg bahnen muss.

Es hat sich nun gezeigt, dass dieses Gerät dank seiner robusten Konstruktion Kerne bis zu $80 \mathrm{~cm}$ Länge liefert; bei Verwendung des schweren Kopfteils als Ramme sind auch noch grössere Längen möglich. Auch kann es anstelle von normalen Plexiglasröhren mit einem Satz aus Rohrringen versehen werden, welche durch eingedrehten Falz ineinandergreifen. Für gewisse Bedürfnisse, z. B. Entnahme von Mikroproben der Sedimentoberfläche oder von sekundären Teilproben mit dünnen Bohr-

1) Die Arbeit wurde an der Abteilung Limnologie der Eidgenössischen Anstalt für Wasserversorgung, Abwasserreinigung und Gewässerschutz (EAWAG) ausgeführt. 
loten usw. hat sich dieser Rohrtyp als sehr hilfreich erwiesen. Längsgeschlitzte und mit wasserfestem Selbstklebeband wieder zusammengefügte Röhren erlauben, den Sedimentkern längs aufzuschneiden [2].

Leider verursacht der Fuss aber trotz seiner minimalen Projektionsfläche manchmal Störungen. So ist die Peripherie der Sedimentoberfläche selten völlig ungestört. Zudem ist die oberste, meist filzartige Schicht bisweilen in einzelne Schollen zerbrochen. Im aufgeschnittenen. Kern ist namentlich in den jüngsten, wasserreichen Schichten die äusserste Partie etwas verschleppt. Zwar sind diese Nachteile für manche Bedürfnisse durchaus tolerabel. Besondere Anwendungen, z. B. Experimente mit natürlich gelagertem Sediment, erfordern aber vollständig ungestörte, intakte Proben.

\section{Das Problem der Entnahme völlig ungestörter Sedimentproben}

Das Problem der Sedimentprobenahme ist so alt wie die limnische Sedimentforschung. Einen Beweis dafür, dass keine standardisierte, betriebssichere Methodik existiert, bilden die geradezu regelmässig erscheinenden Publikationen über dieses Thema; die vorliegende Mitteilung macht davon keine Ausnahme.

Die früheren dünnen Bohrlote sind leicht zu bedienen, liefern aber kompaktierte und nur sehr kurze Kerne, da die grosse spezifische Mantelfläche bzw. die entsprechend grosse spezifische Reibung den Kern in der Röhre rasch bremst und zusammen mit der Röhre weiter eindringen lässt. Das Kolbenlot [3], bei dem der Kern durch einen feststehenden Kolben bzw. durch den dadurch erzeugten Unterdruck gehalten wird, erlaubt erstaunliche Kernlängen; beim Einsatz eines Rammgewichtes lassen sich mit einem verhältnismässig leichten Gerät Kerne bis mehrere m Länge stechen [4]. Die oberflächlichen Schichten, für das Studium der jüngeren Entwicklung von Seen gerade die wichtigsten, sind bei diesen Geräten indessen oft zerstört.

Zuverlässig gute Resultate liefert das Bohrlot von ZüLLIG [5], das ohne Ramme arbeitet und den Kern mit einem Kopfventil hält. Die Kernlänge ist aber beschränkt, ebenso die Materialmenge (innerer Rohrdurchmesser $32 \mathrm{~mm}$ ).

Diese fragmentarische Úbersicht lässt erkennen, dass heute eine Reihe von Geräten zur Verfügung steht, die die meisten Wünsche eigentlich befriedigen. Neue Problemstellungen erfordern nun aber grössere Materialmengen aus möglichst dünnen, definierten Strata sowie - für chemische und mikrobiologische Experimente möglichst grosse und möglichst unverletzte Probeoberflächen. Diese Forderungen bewogen uns, das technische Problem der Sedimentprobenahme erneut aufzugreifen.

Zur Konstruktion eines Versuchsmodells waren folgende Beobachtungen wegleitend:

- Schliesseinrichtungen am unteren Rohrende führen oft zu Störungen der Probeoberfläche. Bei sehr grossen Durchmessern kann sich die Störung auf die Randpartie beschränken.

- Bohrlote mit Kopfventilen arbeiten so sicher wie das Ventil, d.h. der Kern geht nur dann verloren, wenn das Ventil nicht dicht hält. Ist der Durchlass des Ventils im Vergleich zum Rohrdurchmesser klein, so ist die Kernlänge gering und die Oberfläche oft gestört. 
Das im folgenden Kapitel beschriebene Gerät hatte demzufolge das nachstehende Pflichtenheft:

- Die Proberöhre darf (im Gegensatz zum Gerät mit Schliessfuss) keinerlei Anbauten, Nocken usw. tragen, nichts, was das Eindringen ins Sediment hindern oder den gestochenen Kern stören könnte.

- In Anbetracht der Tatsache, dass die oberste Schicht des Seesediments äusserst inkonsistent und verletzlich ist, ähnlich einem zu wasserhaltig geratenen Pudding, und der Schnittvorgang deshalb immer kritisch ist, muss das Eindringen möglichst erleichtert werden:

Die Schneide des Rohres hat messerscharf zu sein, und das obere Rohrende muss während des Einstichs voll offen sein, damit kein Rückstau erfolgt, dessen zurückschlagende Turbulenz die Probeoberfläche nachträglich zerstören kann.

\section{Beschreibung des Gerätes}

\subsection{Ohne Verschluss}

Das Gerät ist prinzipiell eine vergrösserte und in Details verbesserte Weiterentwicklung des erwähnten Schlammlotes von ZüLLIG [5]. Es setzt sich (von unten nach oben) aus dem Probenahmerohr (siehe Abb. 1,2), dem aufgesetzten Kopfteil und dem Auslöser zusammen.

Das Rohr besteht aus Plexiglas (Röhm \& Haas $120 / 110 \mathrm{~mm}$; die preisgünstigere Ausführung aus extrudiertem Material "xt" kann ohne Nachteil verwendet werden). Der Fuss ist mit einer Ringkerbe versehen, die in dieser Ausführung lediglich zur Aufnahme der Verschlussdeckel-Bandage dient.

Erfahrungsgemäss gibt der Ủbergang des oberen Rohrendes zum Schliessventil Anlass zu Schwierigkeiten. Dieses Problem wurde hier in der Weise umgangen, dass das Rohr selber die Funktion des Ventilsitzes ausübt (Abb. 4). Der axial bewegliche Deckel (= Ventil) ist drehbar gelagert (Abb. 1,3), was mit einem Kugelgelenk elegant, aber aufwendig zu lösen wäre; die von uns ursprünglich als rasche Improvisation gewählte Befestigung mit Schrauben und einem zentralen Gummipuffer hat sich jedoch einwandfrei bewährt. Allerdings müssen die Bohrungen im Deckel, welche die Schraubengewinde aufnehmen, dicht verschlossen sein (Abb. 2).

Das Rohr wird mit 6 Schrauben, welche in genau passende Sacklöcher (nicht durchgehende Bohrungen) eingreifen, im Kopfteil befestigt. Bruchschäden an den Rohren durch diese Fixierung konnten bisher nicht beobachtet werden; sie passieren nur, wenn die Befestigungsschrauben stark angezogen werden und die dünnwandige Stelle des Rohres dadurch einbricht.

Das obere Rohrende ist plan gedreht und mit feinen Rillen versehen, um mit der Dichtung, welche in einer Nute im Deckel eingelassen ist (Rundschnur aus weichem Silikongummi), einen absolut dichten Verschluss zu gewährleisten. Der Deckel passt sich dank seiner beweglichen Lagerung von selbst an (Abb.4).

Das Schliessventil läuft im Tragjoch in einer kräftigen Führung, auf der eine Seilführungsrolle (sog. Block) aufgebaut ist. Ein zweiter, beweglicher Block ist am oberen Ende der Führungsstange aufgehängt. Sie bilden zusammen einen Flaschenzug, der bei Aufwärtszug des Tragseils den Deckel mit 2:1 übersetzter Kraft auf das Rohrende 
presst (Abb.3). Das eine Ende des Zugseils ist am Tragjoch befestigt, das andere am Auslöser, der in Bereitschaftsstellung (während Fieren und Stich) am Tragring des Deckels eingehängt ist. Nach erfolgtem Stich wird er mit einem Fallgewicht ausgelöst, wodurch der Deckel, den eine schwache Spiralfeder offenhält, durch das Hieven geschlossen wird.

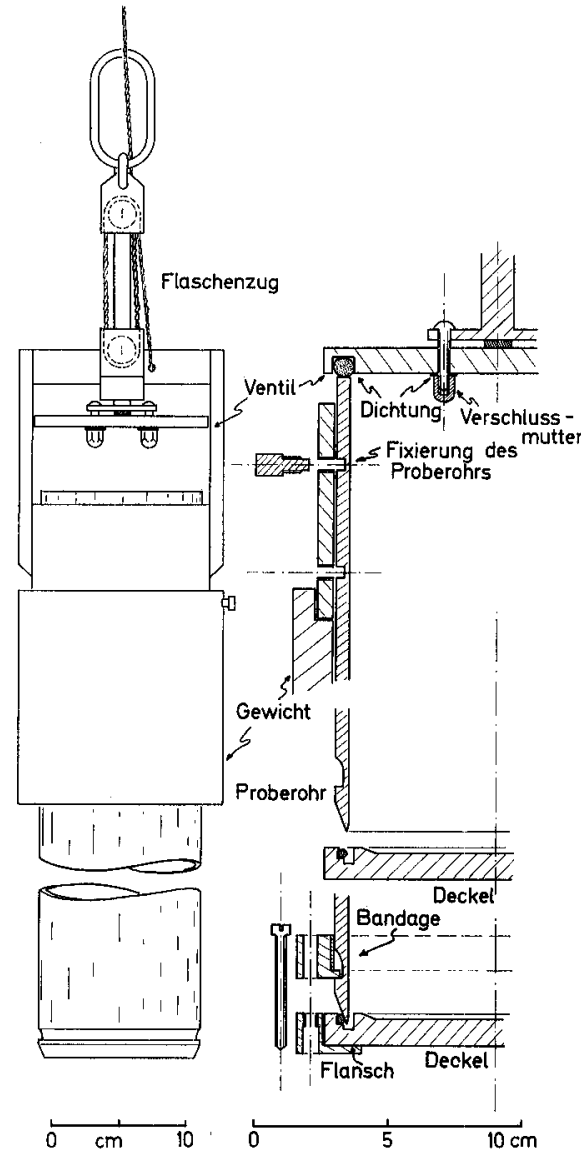

Abb. 1. Schlammbohrgerät, Schema (ohne Verschlussautomatik). Links: Ansicht, rechts: Details im Schnitt.

Fig. 1. Sediment corer, scheme (without automatic closing mechanism). Left: view of apparatus, right: cross-section details.

Als Ausklinker verwenden wir das 1969 beschriebene, aus Stahl gefertigte Modell [1], das sich gut bewährt hat (Abb. 2, 3).

Das ganze Gerät ist mit Ausnahme der Schließschaufeln (Abb. 4) aus Stahl gefertigt, Kleinteile aus Chromstahl und Messing.

\subsection{Handhabung und Verschluss}

Die Entnahme von Sedimentproben erfordert stets grösste Aufmerksamkeit, die sich auch auf die Randbedingungen erstrecken soll. So ist es auch bei ruhigem Wetter unumgänglich, das Arbeitsboot zu verankern. Tiefenkontrolle durch sorgfältige Lotung ist selbstverständlich; Dauerkontrolle durch ein Echolot ist sehr hilfreich. 


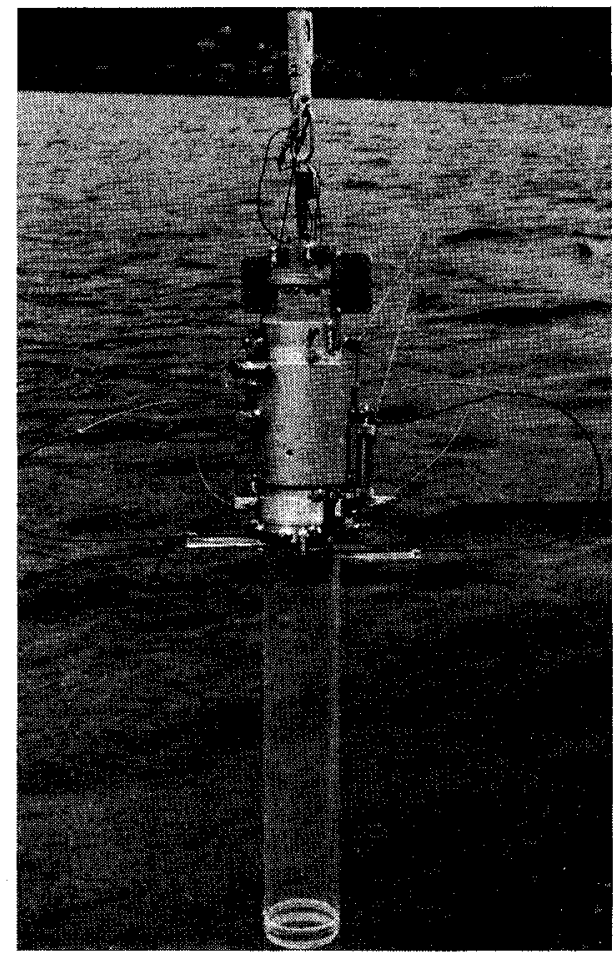

Abb. 2. Bohrgerät inklusive Verschlusswagen, bereit zum Einsatz. Die Leinen für den Wagen und die Bremse sowie die Bowdenzüge für den Auslöser des Gewichtes und die Verschlussplatten werden mit Federarmen abgespreizt bzw. klargehalten.

Fig. 2. Corer with automatic closing device ready for use. The cords for closing device and break as well as the Bowden cables for the release trigger of the weight and the closing plates are kept apart by means of spring holders.

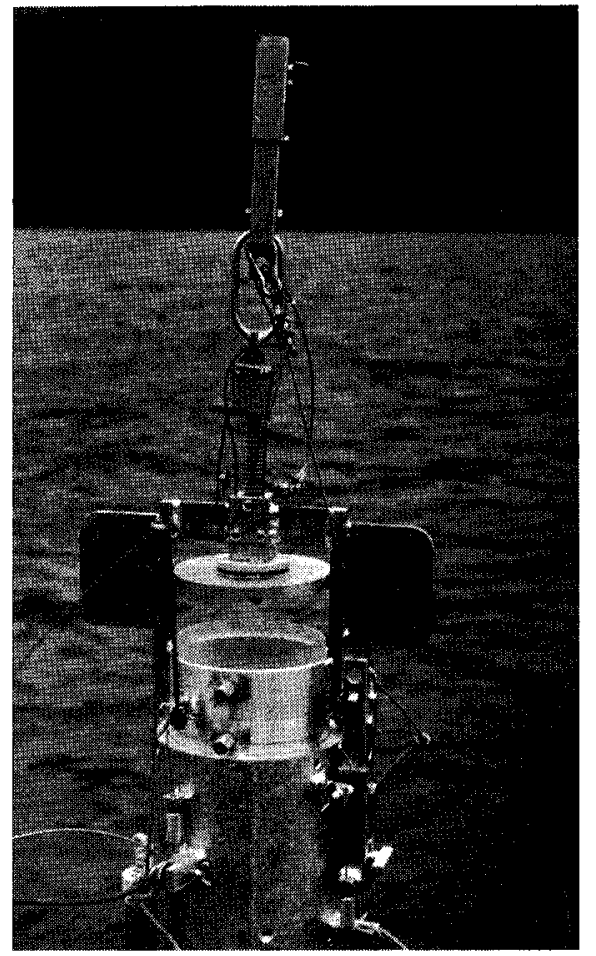

Abb. 3. Aufhängung, Kopfteil und Gewicht. Rechts ist der Ausklinker des Gewichts sichtbar. Unmittelbar über dem Ventildeckel und unterhalb des Tragrings sind die beiden Blöcke des Flaschenzugs sichtbar.

Fig. 3. Suspension, headpiece and weight. The release trigger of the weight is visible to the right. The two pulleys of the block-andtackle are visible directly above the valve cover and below the cable ring.

Das Gerät wird bis rund $2 \mathrm{~m}$ über Grund abgesenkt und dort einige Minuten ruhig hängen gelassen, um Pendelbewegungen ausklingen zu lassen. Dann wird es mit der Seilwinde mit 0,5 bis $1 \mathrm{~m} / \mathrm{sec}$, also zügig, aber kontrolliert, in das weiche Sediment eingesenkt. Mit einem Fallgewicht wird das Ventil freigegeben. Das Gerät wird sodann unverzüglich aus dem Sediment herausgezogen und hochgehievt. Es wird langsam über die Wasseroberfläche hochgezogen, kurz kontrolliert, ob die Probe den Anforderungen entspricht und möglichst, solange das untere Ende noch im Wasser ist, mit einem Deckel verschlossen. Dieser Deckel (Abb. 1) wird von der Seite herangeführt, um den allenfalls unten heraushängenden Schlammzapfen damit abzustreifen. Ein Verschlussflansch (Abb.1) wird von unten her auf den Deckel 
aufgesetzt und das Ganze im Boot abgestellt. Bei diesen Manipulationen sind Drehbewegungen zu vermeiden; sie können, da die Wassersäule diese Bewegung nicht mitmacht, wohl aber der Kern, dessen Oberfläche sehr leicht zerstören. Hingegen ist der gestochene Kern erstaunlich unempfindlich auf Neigung des ganzen Gerätes, solange das Rohr vollständig mit Wasser gefüllt und der Ventildeckel geschlossen ist. Sobald das überstehende Wasser eine grössere Bewegungsfreiheit hat, ist grösste Vorsicht geboten. Eine unbedachte Bewegung, und die Kernoberfläche ist wertlos geworden.

Ein Mann sorgt im folgenden für die vertikale Lage und gleicht bei Schwankungen des Bootes allzustarke Bewegungen aus. Der zweite Mann legt die Verschlussbandage an, verschraubt diese und befestigt den Flansch mit den vorgesehenen drei Schrauben daran (Abb. 1). Alle Schrauben werden angezogen; der Deckel hält jetzt dicht. Am oberen Ende werden nun die sechs Halteschrauben gelöst, und das Kopfstück kann vom Rohr abgehoben werden. Das überstehende Wasser soll ungetrübt und die Oberfläche der Sedimentprobe intakt sein. Je nach Verwendungszweck kann das Wasser mit einer Pumpe abgesogen werden; soll der Kern nachher transportiert werden, so ist dies notwendig.

\subsection{Mit automatischem Verschluss}

Sehr weiches, wasserhaltiges Sedimentmaterial kann so inkonsistent sein, dass es sich, während die Probe aufgehievt wird, unter der Schwerkraft deformiert und axial ausfliesst. Aber auch unter günstigen natürlichen Voraussetzungen kann dieser Fall eintreten. Sedimentmaterial aus grösseren Seetiefen oder mit sehr grosser Bioaktivität setzt infolge der Druckentlastung allmählich Gas $\left(\mathrm{N}_{2}, \mathrm{CO}_{2}, \mathrm{NH}_{3}\right.$ u.a.) frei. Nach einigen Stunden können die Gasblasen ein Volumen von mehreren $\mathrm{cm}^{3}$ erreichen. Beim Hochsteigen in der Probe schädigen sie die Lagerung des stratifizierten Materials. Sind die Sedimentproben für eine längere Standzeit (Experimente) bestimmt, so empfiehlt es sich daher, die Kerne dem Experiment entsprechend kurz zu halten. Allerdings wird dann das Material am Rohrende so inkonsistent sein, dass es den Transport bis zur Wasseroberfläche nicht ungestört übersteht.

Um dies zu verhindern, ist ein unterer Verschluss erforderlich, der das Ausfliessen des Kerns verhindert. Da das Material als Ganzes nicht nachrutschen kann, solange von unten her keine Drainagemöglichkeit besteht (welche den Kern ohnehin sofort zerstören würde), ist auch kein dichter Verschluss nötig; ein lose schliessender $\mathrm{Me}$ chanismus genügt.

Die ursprünglichen Bedingungen, vor allem ein glattes, scharfes Rohr, bleiben hier freilich dieselben. Ein Verschlussmechanismus, der am unteren Rohrende fixiert wäre, kommt daher nicht in Frage. Einzig mögliche Lösung ist ein Verschluss, der am oberen Rohrteil in Bereitschaft steht und erst nach der Probenahme und nach erfolgtem Hieven aus dem Seegrund ans untere Rohrende fährt und dann in Funktion tritt.

\subsection{Die Funktion des Rohrverschlusses}

Ein Rohrstück, das mit 6 Rollen auf dem Probenahmerohr fahren kann, trägt 2 symmetrische Verschlussplatten. Diese sind, durch Federn offengehalten, nach aussen abgespreizt (Abb. 4). Diese Verschlussplatten, am Körper des Wagens 


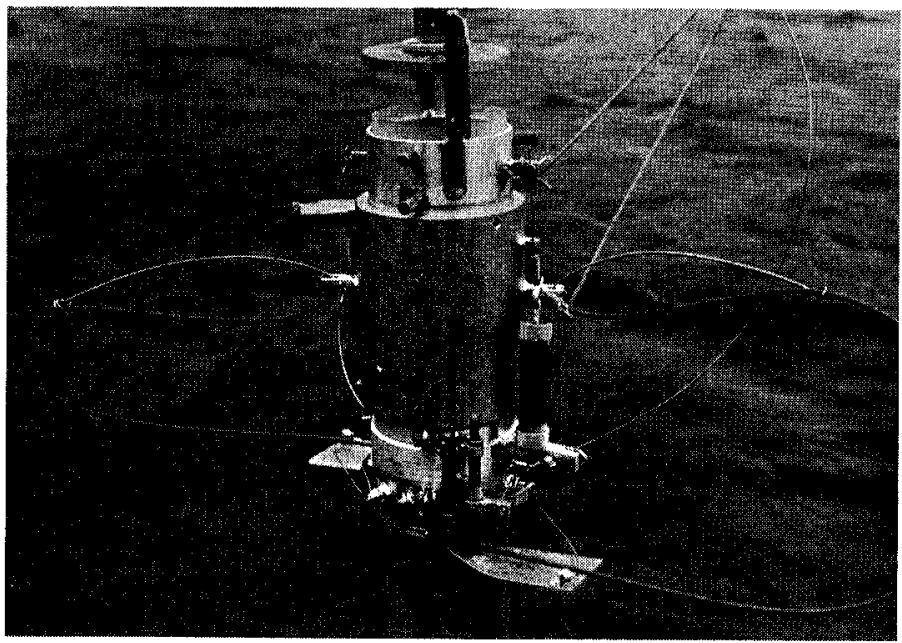

Abb. 4. Kopfteil und Verschlusswagen: Oben links Sicherung des Gewichtes. Am Verschlusswagen ist der Auslösemechanismus zur Betätigung des Gewichtes (= Schliessbewegung) zu erkennen.

Fig. 4. Headpiece and closing mechanism: the weight is secured on the upper left side. Note the release trigger for the weight (closing movement) on the closing mechanism.

in Achsen gelagert, sind über Bowdenzüge mit dem Gewicht verbunden. Dieses ist mit einem Ausklinker am Kopfteil aufgehängt (Abb. 3). Der Wagen ist mit einer Leine zusammen mit dem Tragring des Gerätes am oberen Ausklinker eingehängt. Eine Sicherung gestattet, das Gewicht während der Arbeiten ausserhalb des eigentlichen Probenahmevorgangs festzulegen (Abb. 4). Wird nun nach dem Einstich (wie in Variante 3.1) der Ausklinker durch ein Fallgewicht betätigt, so wird nicht nur der Flaschenzug des Ventils freigegeben, sondern auch der Verschlusswagen. Er rutscht nach unten und steht zunächst auf dem Seegrund auf. Wenn das.Gerät aus dem Seegrund herausgezogen ist, wird er weiter bis zum Rohrende fahren, wobei eine hydraulische Bremse (Zylinder mit lose passendem Kolben, Abb. 4, 6) einen allfälligen Schlag auffängt. Kurz vor dem. Ende des Weges fahren zwei sich gegenüber liegende Tastfinger in die Ringnut ein und stoppen den Wagen. Gleichzeitig löst einer diese Taster eine hart gespannte Feder aus (Abb. 4, 6), welche über einen Bowdenzug den Auslöser, mit dem das Gewicht aufgehängt ist, betätigt. Dieses fällt nun und zieht über die erwähnten Bowdenzüge die beiden Verschlußschaufeln zu (Abb. 5, 6). Das Gewicht dient somit in der ersten Phase zur Beschwerung des Gerätes, in der zweiten als Laufgewicht oder Energievorrat für den Verschluss.

\subsection{Demontage des Schliesswagens, Verschluss der Probe}

Um bei dieser Anordnung die Proberöhre unten verschliessen zu können, muss vorerst der Verschlusswagen entfernt werden. In Anlehnung an das frühere Gerät [1] verwenden wir einen Präpariertisch, der erlaubt, den Verschlusswagen gegen einen Deckel zu vertauschen, ohne die Probe zu stören (Abb. 7 und Abb. 8 A bis K). 


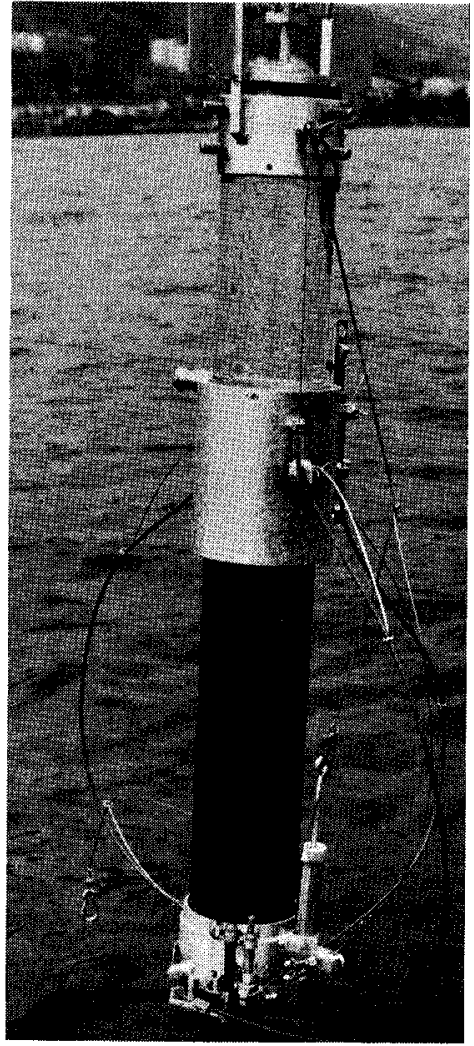

Abb. 5. Nach der Probenahme: Das Gewicht hat die Verschlussplatten über die Bowdenzüge geschlossen und hält sie in dieser Lage. Der Ventildeckel wird durch den Flaschenzug auf das obere Rohrende gepresst.

Fig. 5. After sampling: the closing plates have been closed and are kept in place by the weight on the Bowden cables. The valve cover is pressed against the upper end of the tube by the block-and-tackle.

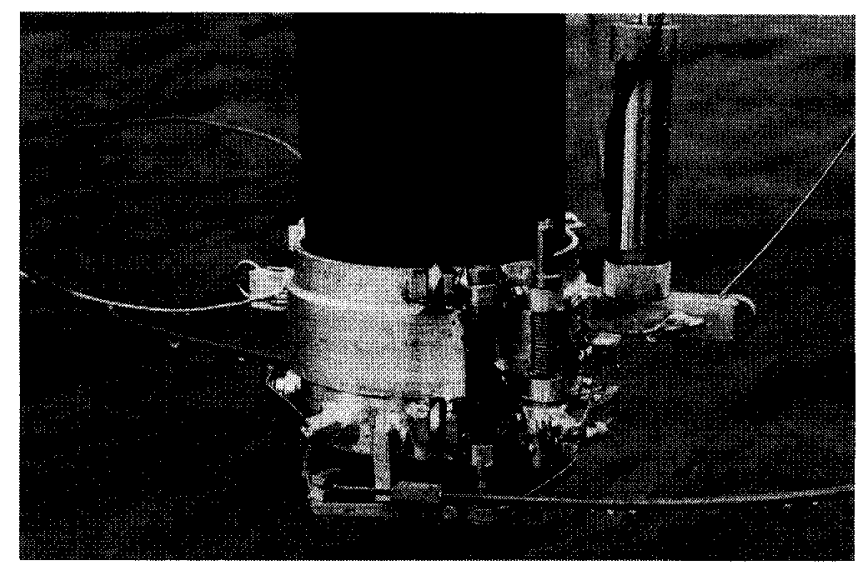

Abb. 6. Detailbild des geschlossenen Verschlusswagens.

Fig. 6. Close-up of the closing mechanism when closed. 
- Das Gerät wird mit den vorstehenden Achsen der beiden Schließschaufeln in höhenregulierbare Auflagen gestellt. Ein Mann hält das Ganze vertikal. Eine Lamelle aus Phosphorbronce (0,2 mm dick) wird zwischen den Schaufeln und der Röhre eingeschoben (A). Sie soll bei den weiteren Manipulationen das Ausfliessen des Materials verhindern.

- Der unter dem Gerät befindliche runde Teller wird in der Höhe so verstellt, dass er die Verschlußschaufeln eben berührt (B).

- Das Gewicht wird in Ruhelage gehoben und gesichert.

- Mit einem Keil von gleicher Dicke wie die Verschlußschaufeln werden diese (unter der Bronzelamelle) auseinander gedrückt und der Keil soweit eingestossen, bis sein paralleler Teil unter dem Schneidfuss liegt (C). Die dabei nötige Drehbewegung ist in Abb. 8 nicht dargestellt, ergibt sich aber in der Praxis zwangsläufig.

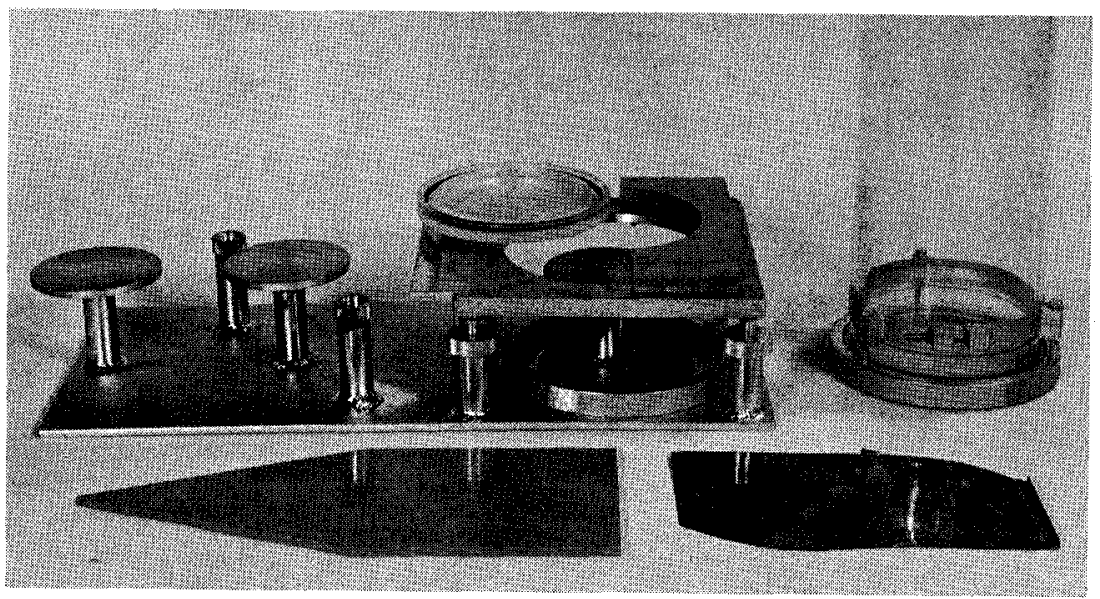

Abb.7. Präparationstisch. Die längsgeteilte Tischplatte ist in den Tischrahmen (rechts) eingesetzt, darauf der Anschlag zur Zentrierung des Rohres, daneben der Deckel. Unter dem Tisch ist ein Flansch sichtbar. Rechts steht eine verschlossene Röhre.

Fig. 7. Preparation table. The lengthwise divided plate is set in the table frame (right), the stop to center the tube is placed on top, the cover follows. There is a fixing flange under the table. To the right a closed tube.

- Die Bowdenzüge werden gelöst. Der Kopfteil wird gelöst und abgehoben, gleichzeitig wird der Verschlusswagen nach oben über die Röhre abgehoben (D). Stehen drei Mann zur Verfügung, so können Kopfteil und Wagen ohne Lösen der Bowdenzüge entfernt und sogleich auf eine neue Röhre aufgesetzt werden.

- Der Rahmen des Tisches wird eingesetzt (E). Auf dem Boden des Tisches wird ein Halteflansch, auf dem zentralen Teller ein Verschlussdeckel bereitgelegt. Die beiden eingeschnittenen Tischhälften, in welche der Deckel genau passt, werden eingesetzt. Sämtliche beweglichen Teile werden in der Höhe justiert. Die Röhre 

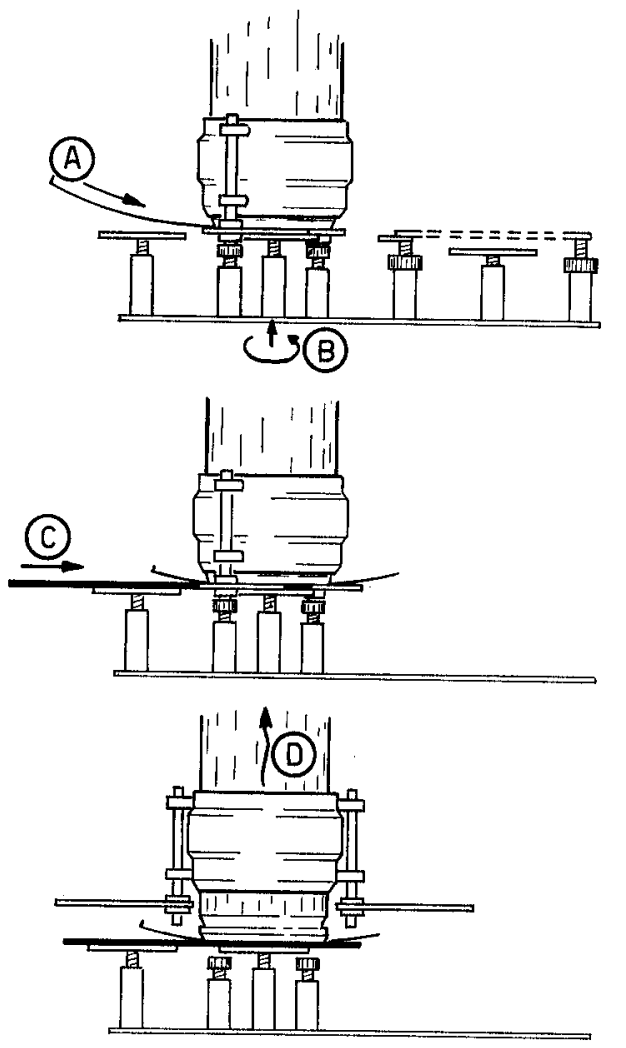

Abb. 8. Präparation der Probe, Schema. Die Beine des Tisches sind in der Zeichnung weggelassen. Erläuterungen siehe Text.

Fig. 8. Preparation of a sample, scheme. The table's legs have been omitted from the drawing. German text gives further details.

wird nun mit dem Bronzeblech auf dem Keil, der auf dem auskragenden unteren Rand des Tisches aufliegt, vorsichtig auf den Tisch hinübergezogen $(G)$. Ein aufsteckbares, halbkreisförmig ausgeschnittenes Anschlagsegment zentriert die Röhre genau über dem Deckel (F).

- Die Bronzelamelle wird unter dem Anschlag herausgezogen. Die Röhre senkt sich mit der Schneide in die mit dem Dichtungsring versehene Ringnut des Deckels $(H)$. Als Dichtung wird ein sog. O-Ring verwendet.

- Keil, Tischplatten und Tischrahmen werden entfernt (I). Der Deckel wird durch Anlegen einer Bandage und Verschrauben mit dem unten bereitliegenden Flansch fixiert (K).

Mit einer neuen Röhre versehen, steht das Gerät nun für die nächste Probenahme bereit. Die ganze Demontage dauert, geübtes Personal vorausgesetzt, eine Viertelstunde. Da der Ablauf an sich logisch ist, ist die Beschreibung komplizierter als der Vorgang selber. 


\section{Erfahrungen}

Mit dem beschriebenen Sedimentstechgerät ist es möglich, in Seesedimenten Proben von einem Durchmesser von $110 \mathrm{~mm}$ und einer Kernlänge von $60 \mathrm{~cm}$ und mehr bei ungestörter Schichtlagerung und unbeschädigter Oberfläche zu gewinnen. Dabei ist folgendes $\mathrm{zu}$ berücksichtigen: Seesedimente sind ein ausserordentlich schwierig zu behandelndes Material. Der Erfolg einer Probenahme hängt zwar wesentlich vom verwendeten Gerät ab, ebensosehr aber auch von der Erfahrung und vom Fingerspitzengefühl der Bedienungsmannschaft. Dasselbe gilt für die Präparation der Proben.

\section{Zusammenfassung}

Es wird ein neues Gerät zur Entnahme von völlig ungestörten Proben aus dem Sediment von Seen beschrieben. Dieses Gerät erlaubt bei einem Durchmesser von $110 \mathrm{~mm}$ eine Kernlänge von $60 \mathrm{~cm}$ und mehr. Technische Bedingungen sind: Proberöhre ohne störende Aufbauten, mit messerscharfer Schneide. Das Kopfventil (zum Halten des Kerns) darf das Wegfliessen des Wassers während des Stechvorgangs nicht bremsen und muss nachher absolut dichthalten. Bei kompaktem (lehmigem) Sediment ist die Probenahme mit offenem Fuss der Röhre möglich. Für stark wasserhaltiges, ausfliessendes Material wird ein selbsttätiger Schliessmechanismus beschrieben sowie ein Gerät zur nachfolgenden Präparation der Probe.

\section{RÉSUMÉ}

Un nouveau carottier qui rend possible une prise d'échantillon sans altération du sédiment des lacs est décrit. La carotte a un diamètre de $110 \mathrm{~mm}$ et une longueur de $60 \mathrm{~cm}$ ou plus. Les conditions techniques sont: un tuyau d'échantillonnage sans étai gênant avec une lame très tranchante. La soupape au bout du tuyau (pour retenir l'échantillon) ne doit pas retenir l'eau au moment de la percée et doit être absolument étanche après. Dans le cas de sédiments compactes (argile) l'échantillonnage peut se faire sans fermer le bout du tuyau. Il y a un méchanisme de fermeture automatique pour des sédiments d'une haute teneur en eau ainsi qu'un appareil pour la préparation de l'échantillon.

\section{VERDANKUNG}

Das Gerät wurde in der Konstruktionswerkstätte der Versuchsanlage Tüffenwies der EAWAG nach den Plänen der Verfasser hergestellt. Sie danken den Herren Dipl.-Ing. H. Burkhalter, Chef der Versuchsanlage, H.-J. Schälchli, Werkstattchef, für die Bereitschaft, die Arbeiten ausführen zu lassen, und für konstruktive Ratschläge, und vor allem Herrn $\mathbf{H}$. Suter, Mechaniker, für die mitdenkende und technisch ausgezeichnete Ausführung. 


\section{LITERATUR}

[1] АмвӥнL, H., Ein Gerät zur Entnahme ungestörter Sediment-Grossproben in Seen. Schweiz. Z. Hydrol. 31, 132 (1969).

[2] Staub, E., Biologisch-stratigraphische Uniersuchungen an jungen Sedimenten des Vievwaldstättersees. Diplomarbeit ETH 1974 (unveröffentlicht).

[3] ZüLLIG, H., Das kombinierte Ramm-Kolben-Lot, ein leichtes Bohrgerät zur vereinfachten Gewinnung mehreve Meter langer, ungestörter Sedimentprofile. Schweiz. Z. Hydrol. 78, 208 (1956).

[4] Sturm, M. und Matter A., Geologisch-sedimentologische Untersuchungen im Thuner- und Brienzersee, Jahrbuch 1972 Thuner- und Brienzersee.

[5] ZÜLLIG, H., Ein neues Lot zur Untersuchung dev obersten Schlammschichten, zur Messung des Sedimentabsatzes und zur Erfassung bodennaher Wasserschichten. Schweiz. Z. Hydrol. 15, 275 (1953).

Anschrift der Autoren:

Prof. Dr. H. Ambühl, Dipl. rer. nat. H. Bührer, EAWAG, Überlandstrasse 133, CH-8600

Dübendorf 